Instituto Internacional de Investigación y Desarrollo Tecnológico Educativo INDTEC, C.A.

DOI: https://doi.org/10.29394/scientific.issn.2542-2987.2017.2.6.2.29-51

OAI-PMH: http://www.indteca.com/ojs/index.php/Revista Scientific/oai

\title{
Alternativas Didácticas de Lectura para el Fortalecimiento de la Calidad Educativa
}

Autores: Carol del Carmen Terán González Universidad Nacional Experimental "Rafael María Baralt", UNERMB carolterang@gmail.com

Trujillo, Venezuela Edith Perdomo de Vieras Universidad Nacional Experimental "Rafael María Baralt", UNERMB gdevieras@gmail.com

Trujillo, Venezuela Yherdyn Jacinto Peña Delgado Universidad de los Andes Núcleo Universitario Rafael Rangel, NURR casahistoriatrujillo@gmail.com

Trujillo, Venezuela

\section{Resumen}

La presente investigación tiene como objetivo fundamental analizar las alternativas didácticas de lectura para el fortalecimiento de la calidad educativa utilizadas por los docentes en educación media general. El estudio se apoya en los referentes teóricos que plantean la lectura, las estrategias de enseñanza y aprendizaje y los elementos que constituyen la calidad educativa. La misma está bajo un enfoque metodológico positivista enmarcado en investigación descriptiva de campo, para esta investigación, se trabajó con los docentes del liceo bolivariano "Antonio Nicolás Briceño" ubicado en el municipio Valera, estado Trujillo en el cual la muestra estuvo conformada por 13 docentes de diferentes áreas de aprendizaje. Sus conclusiones sostienen que la lectura constituye una de las intenciones más preciadas para la formación integral de los estudiantes, por ende, es una herramienta básica que ayuda a la consolidación de la comprensión, análisis y síntesis para el desarrollo del pensamiento. A su vez, es importante debido a que en cada área de estudio del currículo escolar es abordada con énfasis para la enseñanza de sus contenidos desde un contexto pedagógico.

Palabras clave: alternativas didácticas; lectura; estrategias; calidad educativa. 


\title{
Didactic Alternatives of Reading for the Strengthening of the Educational Quality
}

\begin{abstract}
The present research has as fundamental goal to analyze the didactic alternatives of reading for the strengthening of the educational quality used by the teachers in middle education. The study rests on the theoretical modals that raise the reading, the strategies of teaching and learning and the elements that constitute the educational quality. Under a methodological positivist approach placed in descriptive research of field. For this study, authors worked with the teachers of the Bolivarian middle school "Antonio Nicolás Briceño" placed in municipality Valera, Trujillo state on which the sample was formed by 13 teachers of different learning areas. On the conclusions, it was appreciated that reading constitutes one of the intentions most valued to the integral training of the students, that's why it is a basic tool that helps to the consolidation of the comprehension, analysis and synthesis for the development of the thought. In turn, it's important due to the fact that in every area of study of the school curriculum it is approached emphatically for the education of its contents from a pedagogic context.
\end{abstract}

Keywords: didactic alternatives; reading; strategies; educational quality.

Date Received: 06-12-2016

Date Acceptance: 13-02-2017 


\section{Introducción}

La educación es la presentación sistemática de hechos, ideas, habilidades y técnicas a los estudiantes. A través de la historia, ha sido considerada como el recurso más idóneo, el eje rector de todo desarrollo y renovación social. Mediante el proceso educativo se transmiten los valores fundamentales además de la preservación de la identidad cultural y ciudadana; puede afirmarse que es la base de la formación, preparación de los recursos humanos necesarios para promover los beneficios a un país, de esta manera, la escuela se convierte así, en el lugar para la adquisición, desarrollo y difusión de los conocimientos relevantes y el medio para la multiplicación de las capacidades productivas de cada individuo.

De allí que, la lectura se aprende y se obtiene un conocimiento, lo cual, es muy importante para la vida humana, la lectura es como eje central de todo proceso de desarrollo del ser, esto garantiza el éxito académico o estudiantil, de modo que, la lectura permite ampliar conocimiento aprender para responder ante los requerimientos de la actual sociedad.

Por esto, la lectura es un instrumento de aprendizaje que permite lograr una buena educación, en efecto, saber leer es una de las cosas más agradables que existen, porque gracias a ella se integra al niño o niña mejor al entorno familiarizándose con las letras, rótulos, frases, instrucciones y palabras por todas partes, descubrir nuevos mundos, personajes e historias, la lectura es una actividad compleja que parte de la decodificación de los signos escritos y termina en la comprensión del significado de las oraciones además de los textos. Durante esta actividad se mantiene una actitud personal activa, afectiva, puesto que aporta sus conocimientos para interpretar la información, regula su atención, su motivación, genera predicciones y preguntas sobre lo que está leyendo.

Desde este punto de vista, para que los niños(as) alcancen la lectura comprensiva, es fundamental desarrollar los cuatro procesos cognitivos que le 
van a permitir la calidad en la lectura, puesto que cada uno de ellos se complementan. Según Cuetos (2008: 19);

Es evidente que la lectura constituye una de las tareas fundamentales durante la escolaridad, especialmente en los primeros años, ya que, por encima de cualquier otro aprendizaje, el objetivo fundamental de la escuela, es que los estudiantes sean capaces de leer de manera eficiente.

En todo caso, resulta indispensable brindar al estudiante la posibilidad de tener acceso a gran variedad de material escrito, generalmente aquellos que propicien la aproximación efectiva del niño al texto a fin de generar situaciones para realizar una lectura de forma agradable y placentera. En tal sentido, para lograr un acercamiento adecuado del niño a la lectura, se hace determinante implementar estrategias que permitan crear un clima atractivo, así como, motivador que de alguna manera incremente el deseo de comprender y comunicarse.

Por lo tanto, es importante seleccionar cuidadosamente las Estrategias Didácticas a la hora de enseñar, pues ellas, representan un conjunto de acciones planificadas y ejecutadas por el docente con una verdadera intención didáctica, de allí se desprende el buen el buen desempeño de cualquier situación de aprendizaje. Es decir, el uso adecuado de las estrategias empleadas por los docentes puede promover en los estudiantes la adquisición de experiencias significativas específicamente en el desarrollo de la lectura, generando en ellos la oportunidad de utilizar este recurso con toda propiedad en diferentes situaciones comunicativas.

\subsection{Propósito del Estudio}

Analizar las alternativas didácticas de lectura para el fortalecimiento de la calidad educativa utilizadas por los docentes en educación básica. 


\section{Recorrido Teórico}

En el marco de la presente investigación, fundamenta aspectos relacionados con el mismo desde el punto de vista teórico, siendo este el resultado de la selección de aquellos aspectos más relacionados desde el punto de vista epistemológico que orienta el sentido de la investigación, de esta manera se presentan a continuación definiciones conceptuales que sustenta la perspectiva teórica.

\subsection{Lectura}

Resulta de relevante importancia para la educación básica el desarrollo de la Lectura como base para que el estudiante consolide el proceso de aprendizaje. Así lo manifiesta Naranjo, al afirmar;

La lectura ocupa un lugar preponderante dentro de los planes de estudios por ser la base del resto de las asignaturas. Ella constituye una de las principales para la asimilación $n$ de la experiencia acumulada por la humanidad. Su enseñanza coadyuva al desarrollo intelectual y afectivo del estudiante, especialmente en el campo de las lenguas extranjeras donde no solo facilita el acceso al conocimiento científico-cultural, sino que facilita el aprendizaje de la lengua meta (2012: 12).

Según lo expuesto en la cita anterior, se afirma que en la medida de que los docentes brinden a sus estudiantes estrategias que les permitan desarrollo los procesos cognitivos de la lectura, éste puede tener mayor habilidad para apropiarse del lenguaje escrito, pues permite ampliar los conocimientos que finalmente lo llevaran a dominar este lenguaje.

En este orden de ideas, Vived y Molina, señalan:

...que la Lectura es una habilidad esencial para la vida. Poder leer permite el acceso a un conocimiento general y al currículo escolar, ayuda a la resolución de problemas y al desarrollo de las capacidades del pensamiento, ofrece acceso a los libros, a los ordenadores, a los periódicos, etc. (2012: 7). 
De esa manera para desarrollar el proceso de lectura se requiere la utilización de varios procesos cognoscitivos que dan lugar a la comprensión de la lectura así como también, un sentido y significado a los conocimientos logrados, para su acomodación en la estructura del juicio de cada ser humano y lograr la edificación del conocimiento al basarse en tareas abiertas, cercanas al problema que al ejercicio rutinario, favoreciendo la transferencia de los resultados a nuevas tareas a desarrollar por medio del proceso de la lectura.

En la actualidad, el aprendizaje a pesar de su carácter individual y endógeno, se compone además no solo de sus representaciones personales sino también de su actividad social y de la experiencia compartida, donde el estudiante no construye su conocimiento en solitario, ya que requiere de procesos mentales en un momento y contexto cultural y particular dado; al respecto, Díaz y Hernández (2002a: 41), considera que la lectura juega un papel relevante en el proceso enseñanza aprendizaje, pues para los autores se reconoce como "una de las habilidades básicas del ser humano", es decir, es la puerta fundamental del saber y conocimiento, es un medio, enriquecedor para la recreación. Cabe destacar, lo mencionado por Spiro la define la Lectura como:

La actividad caracterizada por la traducción de símbolos o letras en palabras y frases que tienen significado para una persona. Una vez descifrado el símbolo se pasa a reproducirlo, así pues, la primera fase del aprendizaje de la lectura está ligada a la escritura. El objetivo último de la lectura es hacer posible comprender la lectura es hacer posible comprender los materiales escritos, evaluarlos y usarlo para nuestras necesidades (2006: 68).

Lo anteriormente citado, expone la particularidad que tiene la lectura para cada estudiante, en la medida que satisfaga el proceso de lectura en ese orden se pueden satisfacer necesidades propias de cada estudiante, este hecho puede representar para los docentes un reto en cuanto al diseño y 
planificación de estrategias que favorezcan el proceso de lectura al considerar las características e intereses propios de cada estudiante a objeto de perseguir la calidad educativa.

Por otra parte, Dubois (2006: 14), señala que la lectura "es un proceso de interacción entre el pensamiento y el lenguaje y la comprensión en la construcción del significado del texto por parte del lector, de acuerdo con sus conocimientos y experiencias", este hecho representa, la posibilidad de vincular el proceso de lectura con experiencias enriquecedoras desde el punto de vista del aprendizaje para así ampliar la posibilidad de sustentar y consolidar la lectura de manera pertinente con las necesidades tanto contextuales como del estudiante. En investigaciones realizadas por Hernández R, la describe como:

Es un plan general que se formula para tratar una tarea, las estrategias se vuelven menos dificultosa a una labor, ya que la atienden inteligentemente con métodos y con experiencia. Las técnicas y los recursos didácticos, están al servicio de la estrategia, son su perta táctica (2010: 71).

De acuerdo con lo expuesto por los diversos autores, la lectura permite al estudiante participar en procesos sociales de entendimiento que afianza su propia identidad al interactuar en una sociedad específica y compartir con otros su misma cultura. Igualmente, es un medio eficaz para obtener información y adquirir conocimientos, puesto que le permite aumentar su vocabulario y potencialidad de comunicación en el ambiente donde este se desenvuelve contribuyendo a su formación holística.

\subsection{Estrategias Didácticas de Lectura.}

La lectura es un proceso complejo de interpretación del significado de un conjunto de símbolos escritos, el cual permite al estudiante adquirir aprendizajes de todas las áreas académicas, por cuanto permite el acceso al 
conocimiento reconstruido y en consecuencia, su enriquecimiento intelectual.

Es por ello, que desde el inicio de su aprendizaje, el estudiante debe adquirir habilidades que le posibiliten la comprensión del texto escrito, razón por la cual el docente, mediante estrategias didácticas diversas, le facilita el desarrollo de ciertos procesos cognoscitivos como el razonamiento, la reflexión, el juicio y la capacidad crítica de análisis y síntesis para considerar las Estrategias Didácticas, es oportuno mencionar a Dorrego E. y García A. (2001), los cuales definen la estrategia didáctica como; "parte importante del diseño instruccional, la cual comprende el conjunto de eventos, actividades, técnicas y medios instruccionales dirigidos a lograr los objetivos del proceso de enseñanza y aprendizaje", citados por Feo y Martínez (2009: 68).

Se explica, que estas estrategias abarcan las estrategias de aprendizaje y de enseñanza. En esa perspectiva, una estrategia didáctica implica tanto estrategias para enseñar como estrategias para aprender, donde debe existir interacción continua tanto del docente como del estudiante, lo que corresponde a decir la interacción entre las técnicas y las actividades.

Por esta razón, Monereo (2005: 03), afirma que las estrategias didácticas no son un principio ni una actuación, sino un proceso mental proyectado sobre la práctica, de los problemas escolares que se necesitan resolver. En sentido amplio, una estrategia es una representación de proceder maleable y adaptativamente, en la que se parte de las variables contextuales y alterando el proceso, según se vayan modificando dichas variables.

Es por ello que, las disposiciones actuales de la enseñanza, influenciadas por las nuevas tecnologías de la información y la globalización esbozan la necesidad que los docentes enseñen a los estudiantes a tornarse en aprendices autónomos, independientes, autorreguladores, capaces de aprender a aprender. Para lograr esto, el docente debe proponer estrategias didácticas que propicien en los estudiantes la capacidad de reflexionar sobre la forma cómo aprenden y de actuar autorregulando su propio aprendizaje, que 
debe orientar, así como guiar el trabajo de aula con estrategias flexibles además de apropiadas, que ofrezcan la posibilidad de desarrollar habilidades para procesar los contenidos académicos y en este caso muy específico los procesos de lectura.

Así, las estrategias didácticas son una de las piezas fundamentales de los procesos de enseñanza y aprendizaje, puesto que de ella dependen la orientación y la operatividad del proceso, implican una interrelación constante con los demás elementos del diseño de enseñanza, como lo son: los objetivos o competencias a desarrollar, los contenidos, las características, conductas, habilidades de los estudiantes, los medios instruccionales y la evaluación.

Ahora bien, se entiende como estrategias didácticas, según lo expuesto, como aquellos esfuerzos planificados sostenidos y coherentes que buscan que un contenido educativo o un conjunto de ellos sea de acceso a los estudiantes que mediante un esfuerzo de aprendizaje también estratégico logren acceder a este contenido y construir sobre lo dado, adaptarlo, desecharlo o simplemente agregarlo a sus acervos cognitivos.

De lo anteriormente expuesto se deduce que, las estrategias didácticas de lectura, representan un medio para alcanzar la adquisición de conocimientos y habilidades en los estudiantes, por medio de las cuales debe estar presente un proceso comprensivo por parte del lector y deben estar elaboradas para que éste pueda lograr su comprensión. En ese sentido, el estudiante se convierte en un ser que piensa, actúa, crea y expresa todo lo que siente. En ese grado de libertad, irán corrigiendo sus desaciertos con seguridad y paso firme, para adentrarse en el complejo mundo del conocimiento.

\subsection{Estrategias Didácticas de Enseñanza.}

De acuerdo a Díaz y Hernández (2002b), las estrategias didácticas de enseñanza, son los recursos o procedimientos que se utilizan con el objeto de 
facilitar el aprendizaje y comprensión de los estudiantes. Son previamente seleccionadas y planificadas por el docente, en virtud de las características del grupo, del contenido a enseñar, de sus objetivos y de sus propias características personales a fin de garantizar el éxito y el buen desempeño de la misma. Al respecto Mayer (1984), Shuell (1988), West, Farmer y Wolf (1991: 23), definen las estrategias de la enseñanza, como los "procedimientos o recursos utilizados por el docente, para promover aprendizajes significativos", es decir, que son las estrategias que pueden diseñar y utilizar en los estudiantes, que consiste esencialmente en proporcionar una ayuda ajustada a la actividad constructiva de los alumnos.

\subsection{Estrategias de Aprendizaje de la lectura.}

Una estrategia de aprendizaje es una acción que se complementa con la ejecución de un conjunto de pasos o habilidades empleadas por el estudiante en forma intencional, como un instrumento que genera la oportunidad de aprender significativamente y solucionar problemas relacionados con su entorno académico. En este sentido Díaz y Hernández (2002c: 115), afirman: "Las estrategias de aprendizaje son ejecutadas voluntaria e intencionalmente por un aprendiz".

En otras palabras, una estrategia de aprendizaje puede resultar un medio que un estudiante o cualquier persona llegue a utilizar como vía o recurso para llevar a cabo la planificación de cualquier actividad relacionada con la adquisición de un nuevo conocimiento o solución de un problema que de alguna manera les proporcione resultados positivos a los objetivos previstos y estén acordes con sus capacidades y limitaciones.

También para Goodman, (1982: 118), "El aprendizaje de la lectura comienza cuando el niño va descubriendo y desarrollando las funciones del lenguaje escrito, y cómo leer es buscar significados", es decir, para el aprendizaje de la lectura implica un nivel adecuado del desarrollo intelectual y 
social, obligar al niño a leer antes de que esté listo para hacerlo, casi siempre deriva en el fracaso del aprendizaje y en el consecuente daño psicológico al niño.

En este mismo orden de ideas, Díaz y Hernández (2002d: 47), consideran que las estrategias de aprendizaje pueden clasificarse en función de que tan generales o especificas son, del dominio de conocimiento al que se aplican del tipo de aprendizaje que favorecen (asociación o reestructuración), de su finalidad y del tipo de técnicas particulares que conjuntan. En este sentido hacen referencia de algunos autores para establecer la clasificación de las siguientes estrategias de aprendizaje:

\subsection{Estrategias de recirculación.}

De acuerdo con Casamajor, Dussel, Montes, y otros, las estrategias de recirculación:

...se consideran como las primitivas de cualquier aprendiz, son las más simples implica un procesamiento de carácter superficial y son utilizadas para conseguir un aprendizaje al pie de la letra de la información. La más utilizada es el repaso con el objeto de memorizar hasta establecer una asociación que permita retener. (2011: 35).

En este caso, se acude al repaso, el cual radica en repetir una y otra vez la información que se desea recolectar en la memoria hasta adquirir una internalización al pie de la letra. En este caso se pueden utilizar las siguientes actividades propuestas por Serrano y otros (2004a). Trabalenguas, adivinanzas, refranes entre otros.

\subsubsection{Trabalenguas.}

Según Serrano y otros (2004b: 26), "los trabalenguas constituyen un ameno ejercicio a la imaginación y pronunciación de palabras. La tradición oral cuenta con un gran repertorio de trabalenguas que desde hace muchísimo 
tiempo los niños han utilizado para jugar". Asimismo, los niños pueden expresar los trabalenguas que saben, pueden también crear otros nuevos y se les concede la libertad para trabajar en parejas o en grupo, se planifican competencias y al finalizar, los niños y niñas pueden escribir el que sea de su agrado. Estos, ayudan a los estudiantes a una correcta pronunciación cuando algo presentan alguna dificultad en la adquisición de una habilidad de pronunciación. A través de la práctica y ejercicios se puede lograr que los niños lean un poco mejor, sin embargo, para alcanzar la Fluidez Lectora el niño tendrá que aprender a leer grupos fónicos y para ello se les deberá enseñar a interpretar los signos de puntuación.

\subsubsection{Adivinanzas.}

Serrano y otros (2004c), señala que las adivinanzas constituyen una estrategia interesante porque se trata de un juego acompañado de ritmo y sonido que expresa con palabras una invitación al recuerdo, a la agilidad mental y la imaginación. El juego se inicia invitando a los niños a evocar adivinanzas que ya tienen memorizadas y tratar de responder otras. Para realizar esta actividad se utilizan objetos conocidos por los niños, se describen las características y se inventan nuevas adivinanzas. En lo esencial se traduce, que las adivinanzas son estrategias muy valiosas para hacer pensar a los estudiantes; un medio eficaz para fomentar la escritura, la oralidad, la lectura e inclusive la investigación, las mismas, tienen un encanto especial, y a veces resultan píldoras de ingenio y de sabiduría.

De acuerdo con Serrano y otros (2004d), manifiestan que estos estimulan la evocación de imágenes y conocimientos concretos relacionados con algunas situaciones de la vida real. Lo importante es memorizar el refrán, entenderlo y analizarlo para lograr aplicarlo sobre algún acontecimiento específico. se puede iniciar leyendo un texto que utilice refranes y dejar que los niños expresen si lo conocen o no. se les invita también a decir los refranes 
que conocen, que elijan uno e imaginen una situación que pueda justificar dicho refrán. Los estudiantes pueden investigar sobre otros refranes y los traigan al salón para memorizar, compartir y socializar.

\subsubsection{Estrategias de elaboración.}

Considerando lo planteado (Marugán, 2009: 55), la elaboración favorece la generación de nueva información y la recuperación de lo ya aprendido, se pueden establecer diferencias entre elaboración visual y verbal semántica, donde se localizan imágenes visuales y significado de la información que se obtiene a través de una verdadera codificación del contenido. Para canalizar este tipo de estrategias se utilizan las siguientes actividades: resúmenes, idea principal, idea secundaria, entre otras, es decir, la elaboración, es una estrategia de tipo más profundo, supone un papel más activo y constructivo por parte del estudiante.

Dicho de otro modo, con las estrategias de elaboración se van consolidando relaciones significativas entre los conocimientos previos y la nueva información, de ahí su importancia en todas aquellas tareas de aprendizaje donde el sujeto deba reelaborar el material para obtener una auténtica interpretación.

\subsubsection{La idea principal.}

Por consiguiente, Mayo (1997: 47), sostiene que la idea principal es el resumen de un texto o párrafo. Algunos autores lo presentan al comienzo, en el medio o al final. Para localizar la frase principal se busca determinar las palabras que más se repiten, pues allí puede estar la clave de lo que quiere decir al autor, aunque muchas veces esta puede estar sustituida por sinónimos o pronombres, lo relevante es que allí debe estar la esencia del escrito. Es importante destacar que la idea principal es imprescindible en el párrafo y que al suprimirlo, este puede quedar sin sentido. 


\subsection{Calidad Educativa}

Para iniciar el aspecto relacionado a la calidad Educativa se refiere lo planteado por Malpica al señalar;

Calidad Educativa es aquella que se relaciona con la idea de la mejora continua (es decir, en educación no debemos "arreglar" lo que está "estropeado", sino mejorar continuamente lo que hacemos en las aulas, por lo tanto, podemos definir la calidad educativa o pedagógica como la mejora continua de la coherencia entre aquello que se pretende de quien aprende cuando acabe su formación, lo que de verdad ocurre en las aulas para garantizarlo y los resultados de aprendizaje obtenidos. (2013: 56).

A tal efecto, de acuerdo con Ziga;

Existen al menos los siguientes criterios para evaluar la calidad educativa: eficacia, eficiencia, pertinencia y relevancia entre otros. Como cualquier otro sistema educativo, la educación se debe evaluar a través de un enfoque sistémico que contempla criterios de coherencia, eficacia, eficiencia, funcionalidad y equidad. (2001: 25).

Con referencia a lo expuesto por los autores, una educación de calidad debe permitir a los individuos alcanzar metas importantes como la adquisición y apropiación de conocimientos, desarrollo de habilidades que le permitan desarrollarse como persona, ser productivo, convivir, interrelacionarse con su ambiente y contexto y ser gestor de su propio aprendizaje, Pérez (2013: 87); El gran desafío de la educación venezolana es aumentar la calidad, de modo que proporcionemos a todos los estudiantes las competencias esenciales productivas y ciudadanas para que puedan incorporarse activamente en la sociedad y ejercer sus derechos esenciales.

Para poder desarrollar prácticas pedagógicas efectivas y eficientes, es necesario tener muy bien desarrollados los hábitos de lectura tanto el docente como los estudiantes. Mientras más desarrollados se tengan los hábitos de lectura en una sociedad, mayor será el nivel de calidad educativa que posea. 
La lectura es un hábito que debería formar parte de la vida de toda persona. Es difícil pensar en una actividad que no requiera de ninguna forma la necesidad de la lectura. Obviamente, en algunos ámbitos la necesidad es mucho mayor, tanto que no sólo es necesario, sino imprescindible, tal es el caso del ámbito escolar. Tal es el caso planteado por, la UNESCO (2008: 10), sobre el concepto de calidad de la educación la base de la misión de la educación como derecho humano fundamental, conformado por cinco dimensiones esenciales y altamente relacionados: equidad, relevancia, pertinencia, eficacia y eficiencia a fin de contribuir de manera más acertada a la evaluación de la calidad de la educación.

En este caso es necesario señalar, que para la presente investigación se consideran los elementos Pertinencia, Eficiencia y Eficacia, entorno aspectos relacionados con el fortalecimiento de la Calidad Educativa desde la utilización de Estrategias Didácticas de la Lectura, puesto que, la influencia que ofrece la lectura de un texto en ser social es innegable, por lo que crear nuevas y variadas incentivas en la enseñanza y el aprendizaje de la lectura concibiéndola como un objeto del conocimiento, capaz de propiciar las clases para un entendimiento y comprensión de la sociedad y la cultura, a fin de fortalecer la calidad educativa.

\section{Recorrido Metodológico}

La presente investigación, es de tipo descriptiva, que según Rodríguez y Pineda (2003), buscan caracterizar, precisar o determinar condiciones 0 características concurrentes en el hecho o problema. En esta investigación se describieron y analizaron una serie de datos que consistieron en abordar de manera objetiva el uso de alternativas didácticas de los docentes relacionados en la enseñanza de la lectura de los niños de Educación Básica, a fin de obtener una visión concreta que permita establecer una variedad de recomendaciones dirigida a favorecer el proceso de aprendizaje del niño por 
medio de acciones específicas que eleven la calidad de la labor educativa y minimizar la problemática presentada. El Diseño de esta investigación, propios de los estudios de campo, no es experimental, transeccional descriptivo, en tal sentido Hernández, Fernández y Batista (2010: 75), expresan; “los diseños no experimentales se aplican al estudio que se realiza sin manipulación deliberada de variables y sólo se observan los fenómenos naturales para después analizarlos. Asimismo, se trabajó con los docentes del liceo bolivariano "Antonio Nicolás Briceño" ubicado en el municipio Valera, estado Trujillo en el cual la muestra estuvo conformada por 13 docentes de diferentes áreas de aprendizaje se trabajó con los docentes del liceo bolivariano "Antonio Nicolás Briceño" ubicado en el municipio Valera, estado Trujillo.

\section{Resultados}

Se puede afirmar con certeza que el aprendizaje de la lectura va más allá de un simple cambio de conducta, conduce a un cambio en el significado de la experiencia, la cual, a su vez servirá para obtener nuevos conocimientos y nuevos retos. No obstante, el estudiante no construye el conocimiento solo, sino a través de la mediación e interacción con los docentes, compañeros de aula, su comunidad, su globalidad, en un contexto cultural particular, por eso, es tan importante que el docente entienda la influencia que tiene su actuación en el aprendizaje del estudiante (a), sobre todo la manera o las estrategias que el utiliza para impartir sus clases.

Es necesario acotar que la lectura constituye una de las intenciones más preciadas para la formación integral de los estudiantes, por ende, es una herramienta básica que ayuda a la consolidación de la comprensión, análisis y síntesis para el desarrollo del pensamiento. A su vez, es importante debido a que en cada área de estudio del currículo escolar es abordada con énfasis para la enseñanza de sus contenidos desde un contexto pedagógico. 
Igualmente, la visión de la lectura desde la perspectiva informativa permite al lector conocer un ámbito específico del conocimiento; desde la perspectiva lúdica que considera como un viaje imaginario y la función formativa que permite construir la personalidad, crecer y cultivar los sentimientos de los estudiantes. Por ello se debe reconocer estas funciones en el acto de leer como elemento vital, debido a que su entorno se encuentra rodeado de escrituras que lo envuelve en diferentes géneros tales como el ensayo, la poesía, la novela y el cuento, que despliegan una misma función; dialogar con el lector.

Al Categorizar los elementos de la calidad educativa con respecto a la frecuencia de uso de los indicadores, pertinencia, eficiencia y eficacia, se indagó que los docentes encuestados están siendo poco eficaces al promover y utilizar este tipo de estrategias. Esto demuestra, que el docente no está habitualmente inclinado a utilizar estrategias que consoliden significativamente el aprendizaje de los estudiantes.

\section{Conclusiones}

Se pudo establecer que los docentes desarrollan un proceso nada efectivo en torno a la utilización de estrategias didácticas de la lectura al promediar los resultados, considerando, que la calidad tiene que estar conjugada con la pertinencia y el impacto en un contexto real global y multidimensional, pues no se puede concebir una institución educativa de calidad que no sea pertinente con su entorno social, de manera que para hacer relevante este hecho, es necesario alcanzar resultados académicos exitosos, donde los conocimientos que el estudiante previamente son factores claves a la hora de consolidar el proceso de lectura.

Entre algunas de las alternativas que se sugieren están las siguientes: Ejecutar lectura rápida, esta estrategia selectiva tiene como propósito analizar un texto muy rápidamente en forma global para tomar de él algunos elementos, 
resulta útil para buscar informaciones específicas en un texto que trata de varios temas u observar la importancia del mismo. Seguida de la técnica del "salteo", es leer a saltos fijándose en lo más relevante; revisando de arriba hacia abajo en busca de claves, pistas que se unen y conforman una idea tomando en consideración el conocimiento previo

Utilizar el análisis estructural de un texto, para comprender y captar el texto con mayor facilidad dividirlo en unidades de lectura a las que se debe enfatizar por separado. Estas unidades son extensas o cortas de acuerdo con la capacidad del estudiante para desarrollar una idea, el volumen de información presente y el tipo de texto de que se trata.

Poner en práctica la lectura crítica, consiste en distinguir los hechos de las opiniones, comprender los objetivos del autor, valorar la confiabilidad de las fuentes de información es decir, hacer una crítica al autor en la cual está inmerso el punto de vista del lector y si realmente satisface la necesidad del lector por ampliar o desarrollar su conocimiento, básicamente influye la expectativa y el nivel de conocimiento que el lector posee, así como las tendencias que influyen en su cultura y preparación académica.

Elaborar la lectura reflexiva o comprensiva, representa el máximo nivel de comprensión lectora y consiste en repasar una y otra vez sobre los contenidos objeto de estudio para incorporar nuevas ideas que hayan pasado desapercibidas, tratando de interpretarlos. Es pertinente acotar que este tipo de estrategia es la más lenta debido al nivel de exigencia que contiene el propósito en sí. Para usar esta estrategia es necesario buscar en el diccionario todas las palabras cuyo significado no se posee por completo, aclarar dudas con ayuda de otro libro, atlas, enciclopedia, libro de texto; entre otros.

\section{Referencias}

Casamajor, A., Dussel I., Montes N., Pogré, P. (2011). Formar docentes para

la equidad: II. Editorial Teseo, 332 páginas. 
Cuetos, F. (2008). Psicología de la Lectura. Educación Infantil y Primaria. Séptima Edición. Madrid España. De Cádiz España.

Díaz, F. y Hernández, G. (2002a,b,c,d). Estrategias para un aprendizaje significativo. McGraw-Hill. México, D.F. ISBN: 970-10-3526-7. Recuperado de:

https://jeffreydiaz.files.wordpress.com/2008/08/estrategias-docentespara-un-aprendizaje-significativo.pdf

Dubois, M. (2006). La comprensión de la lectura. Caracas: Editorial Romor. Feo, R., y Martínez, J. (2009). Los Materiales Instruccionales Impresos, pasos para su Diseño. Instituto Pedagógico Experimental Libertador. Miranda, Venezuela. 73 páginas. Recuperado de:

https://www.researchgate.net/publication/306091121 LOS MATERIAL ES INSTRUCCIONALES IMPRESOS PASOS PARA SU DISENO

Goodman, K. y Goodman, F. (1982). Los desaciertos en el aprendizaje de la Lectura. Serie de ensayos no. 5. Ed. IPSE, Buenos Aires.

Hernandez, R. (2010). Utilización de Recursos Didácticos. Departamento de Didáctica y Organización Escolar. Facultad de Educación. Universidad Complutense de Madrid, España.

Hernández, S. R., Fernández, C. C., Baptista, L. P. (2010). Metodología de la investigación. Edición: 5a ed. México: McGraw-Hill / Interamericana.

Malpica, F. (2013). Calidad de la Práctica Educativa. Colección Ideas Clave. Editorial Grao. Barcelona.

Marugán, M. (2009). Importancia de las estrategias generales de aprendizaje en el rendimiento escolar. Quaderns Digitals: Revista de Nuevas Tecnologías y Sociedad.

Mayer, R. (1984). Aids to text comprehension. Educational Psychologist (19) 1 ; 30-42.

Mayo, L. (1997). Actitudes Hacia la Lectura y Niveles de Comprensión Lectora. Editorial Trillas. México. 
Monereo, C. (2005). Podemos aprender a aprender. [Documento en línea]. Recuperado de:

http://www.educaweb.com/noticia/2005/12/12/podemos-aprenderaprender-es-decir-poner-marcha-estrategias-adquirir-898/

Naranjo, S. (2012). Didáctica de la Lectura: Fundamentos, conceptos, habilidades, ejercicios y métodos para la enseñanza de la lectura. Editor EAE.

Pérez, A. (2013). El desafío de la calidad educativa. Recuperado de: https://wp.me/p2GlHj-cv

Rodríguez, Y. y Pineda, M. (2003). La Experiencia de Investigar. Editorial Predios. Valencia, Venezuela.

Serrano y otros (2006a,b,c,d). Formación de lectores y escritores autónomos. Editorial Ariel. Venezuela.

Shuell, T. (1988). The role of the student in learning from instruction. Contemporary Educational Psychology (13).

Spiro, W. (2006). Estrategias significativas para el logro de competencias dentro del aula. Editorial Alba. México.

UNESCO (2008). Educación de Calidad, Equidad y Desarrollo Sostenible: Una concepción holística inspirada en cuatro conferencias mundiales sobre la educación, que organiza la UNESCO 2008-2009. pág. 23. Recuperado de:

http://www.unesco.org/education/Synergies4conferences.pdf

Vived, E., Y. Molina, E. (2012). Lectura fácil y comprensión lectora en personas con discapacidad intelectual. Primera Edición. Universidad de Zaragoza. España.

West, Ch., Farmer, J. y Wolf, P. (1991). Instructional design. Implications form cognitive science. New York, EE. UU. Neetham Height, MA. Allyn and Bacon.

Ziga, A. (2001). Calidad educativa y sociedad. Editorial Spam. España. 


\section{Carol del Carmen Terán González}

e-mail: carolterang@gmail.com

Nacida en la ciudad de Valera Edo Trujillo, Venezuela.

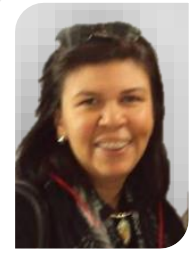
Cursó estudios de Doctorado en Educación en la Universidad Rafael María Baralt, posee Maestría en Docencia para la Educación Superior en la misma universidad, Maestría en Literatura Latinoamericana en la Universidad de los Andes y Licenciatura en Educación Mención Castellano y Literatura ULA-NURR investigadora del Centro de investigaciones literarias y lingüísticas Mario Briceño Iragorry. (CILL) ULANURR, Jefe de la unidad de investigación y proyecto de la Casa de Historia Trujillo, articulista e investigadora con 14 años de servicio en el área de educación y literatura. Ponente en eventos nacionales e internacionales. Docente invitada en pregrado y Postgrado, ULA, UNERMB, UNESR. Coordinadora de la Línea de investigación Memoria, Educación y Discursos emergentes (UNERMB), miembro de la Línea de investigaciones Ciudadanía, hermenéutica y proyectos sociales. (UNERS). Ha realizado artículos en distintas revistas arbitradas del país. 


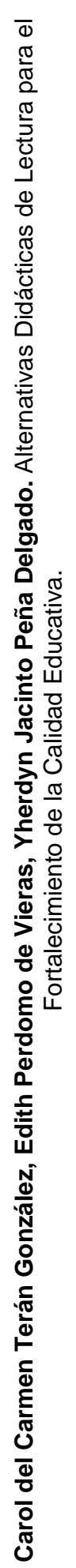

Edith Perdomo de Vieras

e-mail: gdevieras@gmail.com

Nacida en Valera, estado Trujillo, Venezuela. Magíster en Docencia para la Educación Superior Licenciada en Educación Integral Área Concentración Lengua. Doctorante en Ciencias de la Educación. Docente con más de 15 años de experiencia.

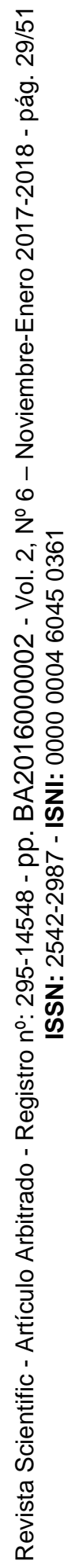

50 


\section{Yherdyn Jacinto Peña Delgado}

e-mail: casahistoriatrujillo@gmail.com

Nació en Trujillo, estado Trujillo, Venezuela. Profesor de

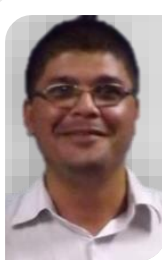

Historia del NURR - ULA. Miembro del Centro de Investigaciones Literaria y Lingüísticas "Mario Briceño Iragorry, Coordinador de La Casa de Los Tratados. Fundador - Coordinador del Taller de Historia "Abya Yala". 